\title{
Ladrones de inocencia: Análisis jurídico-doctrinal de la pederastia y el derecho penal nicaragüense
}

\section{Resumen:}

La pederastia se define como la acción u omisión no accidental, que priva al niño de sus derechos y su bienestar, amenazando y/ o interfiriendo su desarrollo físico, psíquico, social y sexual cuyos autores pueden ser personas, instituciones o la propia sociedad. En la actualidad el fenómeno de pederastia presenta múltiples facetas que inciden de manera negativa sobre la sociedad nicaragüense, causando a su vez. serias afectaciones en la psiquis y en su desarrollo como individuo en los menores.

Uno de los propósitos que impulsaron realizar este ensayo, es problematizar a partir de la sistematización de la información relacionada al tema, e identificar patrones de comportamiento y acciones para su prevención oportuna. Un aspecto fundamental de la investigación, es la diferenciación que se debe hacer entre pederastia y pedofilia, para ello se bizo un breve análisis jurídico-doctrinal de la pederastia. Entre los aportes al tema, está el aprendizaje, la valoración y la acción que podría establecer la norma jurídica ante este tipo de conductas sexuales. En este sentido, se ha querido dar relevancia a las fuentes provenientes de especialistas en la materia y de instituciones encargadas de la protección de la ninery adolescencia.

Palabras clave: pedofilia, pederastia, abuso sexual, transgresión de la ley.

\section{Abstract.}

Pederasty is defined as a non-accidental omission or action that deprive children rights and wellness, threatening and I or interfering their physical, mental, social and sexual development, whose actor can be people, institution or their own society. Actually pederasty phenomenon shows multiple angle that have a negative influence in Nicaraguan society, and also causes severe consequences children development.

This essay aimed to do an analysis and systematization of related documental information, to identify behaviour patterns and actions for timely identification. Through a legal and doctrinal analysis the difference between paedophilia and pederasty was established, which was a fundamental issue of this research. The research contribution are knowledge, assessment and actions to be considered within legal norm to deal this type of sexual behaviour. Important relevance has been given to sources from specialist and institutions responsible to children and adolescent protection.

Keywords: pedophilia, pederasty, sexual abuse, transgression to the legal. 


\section{Introducción}

La Pederastia es un problema universal que ha estado presente, de una u otra manera, en todas las culturas y épocas de la evolución social. Este tipo de prácticas supone una interferencia en el desarrollo sexual evolutivo del niño que puede dejar secuelas muy graves con el paso del tiempo. El psiquiatra alemán Krafft-Ebing (2006) en su obra Psycho-pathia sexual plantea que: Existen diferentes modelos psicopatológicos relacionados con diferentes preferencias sexuales (p. 184). Este criterio sugiere la posibilidad de actividades sexuales de adultos con niños y niñas, generalmente de 15 o menos años de edad. Para que el trastorno se considere como tal, el individuo que lo sufre debe tener más de cinco años que el niño que es víctima, y debe de considerarse la legislación nicaragüense que establece mayoría de edad a partir de los 18 años.

La pederastia es un problema de preocupación a nivel de los familiares y las autoridades nacionales encargadas de velar por la seguridad y bienestar de las personas. Esta acción, transgrede el bien jurídico derivado del art. 3 de la Declaración de los derechos humanos, donde se expresa que: "Todo individuo tiene derecho a la vida, a la libertad y a la seguridad de su persona". En este sentido, toda acción que transgreda y/o ponga en peligro la vida y la seguridad de las personas, incluyendo las agresiones sexuales, caen dentro de la figura delictual, tanto a nivel nacional como internacional. Además de generar rechazo y condena social.

Los delitos sexuales y la acción pederasta, son transgresiones sociales de difícil solución que por su gravedad requiere un abordaje urgente desde diferentes enfoques, tales como socioeducativos, psicológicos y legales. Por tal motivo, este trabajo de carácter documental, realiza un análisis jurídico-doctrinal de la pederastia.

Un primer aspecto que abordará este trabajo, tiene que ver con los conceptos básicos y generales de la pederastia, a partir de lo que sugieren los psicólogos o expertos en la materia. El segundo, hace una breve referencia a la pederastia desde el ámbito jurídico-doctrinal. Por último, se hace un sucinto estudio comparativo, a partir de cada uno de los contextos del derecho comparado, entre los lineamientos jurídicos penales del derecho penal nicaragüense y el derecho penal mexicano, haciendo énfasis en las características, similitudes y diferencias entre ambas legislaciones. Se toman en cuenta también, las diferentes instituciones del Estado encargadas de la protección a la niñez y la adolescencia y las opiniones de los entrevistados especialistas en el tema.

\section{Análisis jurídico-doctrinal de la pederastia: aspectos generales y conceptos básicos}

Percy William (1996) en su trabajo Pederastia y pedagogía en la Grecia arcaica expone que: "En la antigua Atenas, la relación sexual entre un adulto y un púbero se denominaba pederastia, y se consideraba como un elemento más en la relación entre un docente y su discípulo. El amor entre ambos favorecía la transmisión del saber y de las leyes ciudadanas. Por el contrario, el sexo con sujetos pre púberes, denominado anteriormente como pedofilia, era castigado con condenas que podían llegar a la pena de muerte". Así mismo, este autor explica que: "En la Roma antigua, la pederastia estaba muy difundida, pero sin las justificaciones de los griegos, y la pedofilia era también condenada". (pp. 30-35) 
El término Paederastia (pederastia) fue acuñado principalmente en 1886 por el psiquiatra Richard von Krafft-Ebing en su trabajo Psycophatia Sexual. Este autor, la describe como: "El interés sexual dirigido sólo hacia jóvenes pre pubescentes, sin incluir a los adolescentes, un interés que desaparecería con la aparición de los primeros signos de vello púbico”

La pederastia se define desde dos ópticas que no siempre coinciden: la jurídica y la psicológica. Para la académica y jurista Astrid Bracamontes, especialista en derecho penal nicaragüense, la pederastia en el ámbito jurídico, "no tiene una conceptualización específica (...), la ley no usa particularmente la palabra pederastia, sí califica como delito el acceso carnal por vía vaginal, anal o bucal a una persona menor de catorce años, aun cuando no exista fuerza, intimidación o incapacidad de la 100 víctima para defenderse" (Entrevistada, 5 de Septiembre del 2016).

En el ámbito de la psicología, la psicoanalista Tania Bautista considera que "la pederastia es considerada como una parafilia que consiste en la excitación o el placer sexual que se obtienen, principalmente, a través de actividades o fantasías sexuales con niños menores de 13 años y esto es debido a un rasgo multifactorial en la personalidad del que la padece". En este punto, es importante diferenciar la pederastia de la pedofilia. Para José Carlos Fuertes Rocañín (2014) experto en medicina legal-forense, sostiene que pedofilia y pederastia son similares pero no iguales: "Ambas patologías están compuesta de múltiples contenidos semejantes pero esto no las hacen incluyentes entre sî".
Por su parte la psicóloga Magda López expresa que, "Los pedófilos son sujetos enfermos mentalmente hablando, que tienen un trastorno en la elección del objeto sexual y en el control de sus impulsos". Son personas desequilibradas, que con frecuencia sufren por sus impulsos sexuales, que no pueden modular, reprimir, modificar. Siguiendo lo que señalan los especialistas (Fuertes Rocañín y López) una cosa es el enfermo pedófilo, y otra distinta es el pederasta, que siendo un pedófilo pasa a la acción y transgrede la integridad física y psicológica del menor para satisfacer sus abyectos impulsos sexuales. Esto sugiere que todos los pederastas son pedófilos, pero no todos los pedófilos son pederastas.

El pederasta es un delincuente que aunque tiene un trastorno psíquico que limita y condiciona su elección sexual, es consciente de sus actos y en cierta manera podría reprimirlos y controlarlos. Lo que ocurre es que se "deja llevar" por la satisfacción que le origina el contacto sexual con impúberes, dejando a un lado la patología para ser tomada como un acto delictivo.

El manual diagnóstico de los trastornos mentales de la Asociación Psiquiátrica Americana (American Psychiatric Association, APA) describe los siguientes tres rasgos estándar de un pederasta: el primer rasgo es la experimentación, que se da durante un periodo de al menos 6 meses y que consiste en fantasías sexuales intensas o recurrentes, siendo el objeto de atención uno o varios niños pre pubescentes (generalmente, menores de 13 años).

El segundo rasgo es la materialización, que se expresa de diversas maneras. Una de ellas es la atracción erótica que los pederastas sienten por 
los niños la cual no se traduce necesariamente en actos sexuales completos. Sobre este aspecto, la jusrista Eveling Bravo Cabrera, coincide al señalar que la ley califica como delitos de materialización los siguientes acciones: promoción y facilitación de la prostitución de menores; producción o distribución de material pornográfico en los que participen menores de edad; exposición de menores a material pornográfico para lograr excitación y realización de acciones sexuales para procurar su excitación y/o la excitación de otro ante personas menores de edad.

El último rasgo, el lado cognitivo, supone que el pederasta no considera inapropiada su tendencia o conducta, por lo que no suele presentar sentimientos de culpa o vergüenza. La personalidad del pederasta es polimorfa distinguiendo dos grandes grupos: El primero, muestra una inclinación sexual casi exclusiva por los niños y su conducta compulsiva lo hace independiente de su situación personal. El segundo, se caracteriza porque su conducta viene inducida por una situación de soledad o estrés. La psicóloga María Dolores, expresa que los pederastas se manifiestan de la siguiente manera:

Como ansiosos-resistentes, caracterizados por su escasa autoestima que les lleva a buscar constantemente la aprobación de los demás; dado que no consiguen establecer relaciones emocionales con los adultos, se centran en los niños, con los que aumenta su seguridad. En principio, su relación no es sexual, pero la dependencia afectiva puede generarla (...) Los evitadores-temerosos, caracterizados por su gran deseo de contacto con los adultos pero a los que el miedo al rechazo los paraliza. Se centran entonces en los menores y su actitud es poco empática y tienden al uso de la fuerza (...) Los evitadores-desvalorizadores, caracterizados como obsesionados con la independencia y la autonomía emocional; buscan relaciones fugaces e impersonales en las que no es infrecuente el comportamiento coercitivo violento.

En resumen, la pederastia, no es más que una forma de maltrato dirigida al niño o a la niña que se estiman en las edades de 15 años o menos. Esta acción, jurídicamente transgrede un bien protegido y psicológicamente afecta de manera inherente el desarrollo de la sexualidad del mismo.

\section{Análisis jurídico de la pederastia basándonos en el derecho penal nicaragüense.}

En Nicaragua la legislación está determinada por cada entidad y no existe un consenso jurídico sobre la tipificación de esta acción, según lo expresado por Astrid Bracamontes (2016). De lo anterior se puede decir que la valoración jurídica de los delitos sexuales depende del grado de contacto físico entre los órganos sexuales del agresor y la víctima (Cp 2008; arto 172).

Desde el punto de vista jurídico, la pederastia se ha sustentado en figuras jurídicas como la "violación”, el "abuso deshonesto" y el "estupro", establecido en el código penal nicaragüense. Para algunos juristas doctrinales reconocidos en el ámbito del derecho penal, la mera relación sexual entre un adulto y una niña o niño, suele denominarse como: "pederastia", "pedofilia", "infantofilia" y/o "efebofilia". En el lenguaje común es denominada como "abuso sexual" o "agresión sexual de infantes" 
Según Eveling Bravo (2016), la pederastia, en nuestra legislación, no constituye delito alguno, debido a que se encuentra clasificada como una parafilia. Esta jurista afirma que no todas las personas que presentan este "tipo de parafilia son abusadores". Cuando esta inclinación es llevada a la práctica la legislación actúa siguiendo las sanciones penales establecidas.

En el derecho penal nicaragüense no existe un perfil exacto de un abusador pederasta puesto que lo calificado en nuestro código penal es el abuso sexual. Según el tratadista Torres Tópaga (2011), haciendo referencia al delito de violación, plantea que: "es aquel que sancionan las vulneraciones de un bien jurídico, al prever comportamientos que van en contravía del derecho de las personas de disponer de su cuerpo sobre cualquier tipo de actividad sexual" (p.875).

De lo anterior, la naturaleza del delito de violación es la relación controladora del poder individual y social que tiene lugar entre la víctima y su agresor. Según el código penal nicaragüense, "comete delito de abuso sexual la persona que realice actos lascivos, tocamientos lúbricos en otra personas, sin su consentimiento $u$ obligue a que realice haciendo uso de fuerza, intimidación o cualquier otro medio que prive de la voluntad, razón o sentido, aprovechando su estado de incapacidad para resistirse, llegando al acceso carnal u otra conducta prevista en el delito de violación (art.172CP)

Siguiendo la definición del artículo 172 (citado anteriormente) se puede decir, que la violación a menores es el acto mediante el cual una persona tiene acceso carnal con menores de catorce años con fines sexuales, usando fuerza, violencia, intimidación, o cualquier otro medio que prive a la víctima de voluntad, razón o sentido. Desde esta perspectiva jurídica es importante no confundir el delito de violación sexual con otros delitos similares, como el proxenetismo, el acoso sexual y la trata de personas.

En 2015 la Policía Nacional registró 2,314 denuncias de violencia sexual de las cuales el 84\% fueron delitos cometidos contra Niños, Niñas y Adolescentes (NNA) de 1 a 17 años, siendo ésta la edad más vulnerable para sufrir abuso. Otro dato alarmante que sugieren los informes o estudios relacionados, señala que de cada cinco niñas, tres son abusadas sexualmente y de cada cinco niños, dos han vivido abuso. Por otro lado, en el 2015, el Instituto de Medicina Legal (IML) reveló 7,977 peritajes por violencia sexual, lo que sumado a los datos de la Policía ascienden a casi 8,000 NNA que sufren abuso en Nicaragua. Y lo más preocupante es que a inicios de 2016 las cifras van en aumento.

La psicoanalista Tania Bautista (2016) expresa que la situación de la niñez y adolescencia nicaragüense está marcada por dos ejes diametralmente distintos: la del discurso de restitución de derechos y la realidad cotidiana de violencia como la exclusión, la desigualdad social, la precariedad económica y la ausencia de oportunidades de desarrollo. Esta condición expresada por la especialista, es coherente con la situación del país, donde aún existen carencias y/o vacíos en términos de políticas públicas y normativas jurídicas que atiendan temas relacionados con el desarrollo integral de los niños, niñas y adolescentes.

n tal sentido, el Comité de derechos del niño y la niña de las Naciones Unidas en 2013, observó que 13 años después de la entrada en vigencia del Código, todavía no gozaba de las prioridades 
necesarias en Nicaragua, debido a la falta de recursos institucionales, humanos, técnicas y financieras puesto que dificultaba su plena aplicación. Cabe destacar que a partir del debate en torno al tema de la creación de una normativa específica que sancione la pederastia no se concretó nada en la actualidad, quedando pendiente para una posterior discusión.

\section{Lineamientos jurídicos penales: derecho penal mexicano y derecho penal nicaragüense}

El código penal federal mexicano, con las modificaciones del año 2014, en relación al delito de violación sexual, planteó varias situaciones jurídicas interesantes. Una de ellos, el delito de pederastia, fue tipificado en el Código y sus principales disposiciones están enfocadas en la protección y educación de la población mexicana. La figura de la pederastia, aparece contemplada en un título aparte del delito de violación sexual. Es ubicada específicamente en el título octavo denominado: Delitos contra el libre desarrollo de la personalidad (Capítulo VII Pederastia). En su artículo 209 expresa que:

Se aplicará de nueve a dieciocho años de prisión, a quien se aproveche de la confianza, subordinación o superioridad que tiene sobre un menor de dieciocho años, derivada de su parentesco en cualquier grado de tutela, curatela, custodia, relación docente, religiosa, laboral, médica, cultural, doméstica o de cualquier índole y ejecute, obligue, induzca o convenza a ejecutar cualquier acto sexual, con o sin su consentimiento.

La misma pena se aplicará a quien cometa la conducta descrita del párrafo anterior, en contra de la persona que no tenga la capacidad de comprender el significado del hecho o para resistirlo. Si el agente hace uso de violencia física, las penas se aumentarán. Además de las anteriores penas, el autor del delito perderá, en su caso, la patria potestad, la tutela, la curatela, la adopción, el derecho de alimentos y el derecho que pudiera tener respecto de los bienes de la víctima, en términos de la legislación civil. Cuando el delito fuere cometido por un servidor público o un profesionista en ejercicio de sus funciones o con motivo de ellas, además de la pena de prisión antes señalada, será inhabilitado, destituido o suspendido, de su empleo público o profesión por un término igual a la pena impuesta (Cpf.)

El legislador mexicano no dice taxativamente de sostener acceso carnal sino de "quien" (persona o individuo) realice cualquier acto sexual con una persona menor de edad. En otras palabras, una persona menor de dieciocho años, entendiéndose como una forma de acto sexual, además del acceso carnal, la experimentación y/o materialización expresada anteriormente.

Una de las principales característica del artículo 209 es que si existiese entre la víctima y el victimario una referencia que aluda cierta interacción entre ambos, por tanto la existencia de un grado de confianza daría pie a una mayor o menor sanción. La sanción puede ser: prisión, pérdida de obligación del derecho que tenga el victimario con relación a la víctima; la inhabilitación, suspensión o destitución del empleo o profesión.

Por su parte la legislación penal nicaragüense, no contempla esta figura si no que se tipifica el deli- 
to de violación sexual y, lo incluye en el artículo 172 (primer y tercer párrafo) del código penal en calidad de agravante específica. De igual manera, como sanción para este acto criminoso se encuentra la prisión o pena máxima. A su vez la ley 870 o Código de la Familia de la República de Nicaragua en su artículo 295 inciso C y D, señala lo siguiente:

Habrá pérdida de autoridad parental, c) Cuando se someta al hijo o hija a maltratos físicos, psíquicos o morales que lesionen su integridad física y d) aquel padre o madre que promueva para beneficio propio o de una tercera persona, la explotación sexual del hijo o hija mediante tráfico, trata o pornografía, actos sexuales remunerados y turismo sexual infantil (arto 295).

Este artículo manifiesta claramente una forma denominada por la doctrina en materia penal al delito de violación sexual, siendo un delito donde el sujeto activo está determinado. La legislación mexicana también contempla como pieza probatoria fundamental para determinar el daño ocasionado al desarrollo de la personalidad de la víctima, la prueba pericial de los dictámenes médicos (art. 209 Termino del código penal federal mexicano 2014).

Por otra parte, el código penal federal mexicano en su título decimoquinto, tipifica los delitos contra la libertad y el normal desarrollo psicosexual, estableciendo en su capítulo I los delitos de hostigamiento sexual, abuso sexual, estupro y violación. En su artículo 265, describe el tipo penal de violación en los siguientes términos:

Comete el delito de violación quien por medio de la violencia física o moral realice cópula con persona de cualquier sexo, se le impondrá prisión de ocho a veinte años. Para los efectos de este artículo, se entiende por cópula, la introducción del miembro viril en el cuerpo de la víctima por vía vaginal, anal u oral, independientemente de su sexo. Se considerará también como violación y se sancionará con prisión de ocho a veinte años al que introduzca por vía vaginal o anal cualquier elemento o instrumento distinto al miembro viril, por medio de la violencia física o moral, sea cual fuere el sexo del ofendido (Arto 265, Cpf.)

El artículo bajo análisis presenta características interesantes, entre las que podemos señalar:

1) Dentro de los sujetos activos de este delito, se encuentra en primer lugar al hombre. Al describir en qué consiste la cópula, explica en primera instancia la introducción del miembro viril. Esto independientemente que al inicio del artículo se plantea un sujeto activo indeterminado, al incluir el pronombre "quien".

2) La presencia de la violencia física o moral, igual se maneja como en la legislación penal nicaragüense. Este artículo (265) se asemeja al 172 del código penal nicaragüense, con algunas variantes, como por ejemplo, el rango de edad en uso es de menor a catorce años y no se establece la expresión miembro viril.

Se puede enfatizar que el Derecho federal Penal Mexicano está preparado antes situaciones diversas correlacionados con la pederastia. Esto demuestra que México tiene interés por prevenir, atender y erradicar los problemas asociados con este delito sexual. 


\section{Recomendaciones}

La pederastia es una problemática alarmante que atañe a todos a nivel nacional, pero es un tema amplio del que aún hace falta investigar. Todo niño puede ser víctima de esta parafilia, independientemente de su género, físico, trasfondo económico, familiar y/o social; al igual que cualquiera puede ser el perpetrador.

Lo anterior es de vital importancia, puesto que se debe trabajar en la prevención, la sanción y las secuelas que el abuso sexual en cualquiera de sus tipificaciones deja en las víctimas. Sí bien, toda conducta sexual inapropiada (al margen de las normas jurídicas o morales) tiene un origen multifactorial, es deber de las instituciones públicas o privadas, brindarle a atención a los afectados.

El abordaje de la pederastia debe hacerse desde distintas perspectivas. Estudiando la prevalencia de este tipo de violencia con el fin de dirigir de manera efectiva las medidas preventivas, atendiendo los riesgos y sancionando a los infractores. La pederastia es un problema social y de las familias nicaragüenses, siendo ellas los primeros sujetos en la ejecución de la puesta en marcha de planes o normativas que contribuyan al resguardo de los sectores vulnerables (los niños y niñas) a estas prácticas.

Como se ha señalado en este trabajo, la formulación y aplicación de las leyes que protegen a los niños, niñas y adolescente, es de gran importancia para acciones afirmativas sobre el tema del abuso sexual. Además de la ausencia de una estrategia comunicacional que oriente a la población sobre los mecanismos o instancias para la protección o denuncia de casos de abuso, tomando en cuenta que muchas leyes o normas no se aplican o no llenan todo el espectro de problemas que encierra estos delitos.

Si bien existe un cuerpo de leyes (desde la Constitución Política de Nicaragua, código penal, el código de familia y el código de la niñez y adolescencia), convenios, y normativas entre otras, de carácter administrativo las debilidades en su aplicación ocasionan que los pederastas no sientan temor al cometer este tipo de infracciones a la ley. Para disminuir la pederastia y otros abusos sexuales en menores de edad se sugieren las siguientes acciones:

- Brindar información a la población incluyendo a los niños, niñas y adolescentes haciendo énfasis en las sanciones estipuladas en la norma penal.

- Empoderar a la población del procedimiento a seguir para denunciar todas las situaciones que conozcan de abuso.

- Fortalecer las capacidades de los funcionarios públicos para que los procesos no se caigan y así fortalecer la confianza de los ciudadanos respecto al sistema de justicia penal.

- Exigir la garantía de confidencialidad de la denuncia y la protección de la víctima, denunciante y testigo, para de esta forma lograr que la población denuncie

- Diseñar y difundir de manera amplia, campañas informativas sobre los tipos de abusos y las formas en que se expresan.

- Realizar campañas utilizando los canales para mejorar, completar o aplicar las leyes atendiendo el espíritu de la constitución, de igualdad y equidad ante la ley. 
- Proponer un estudio a fondo con el fin de profundizar en el tema de la Pederastia y la importancia de su tipificación en nuestro código penal.

\section{Bibliografía}

-American Psychological Association (2010). Manual diagnóstico de los trastornos mentales (3era ed.) (Trad. M. Guerra Frías). México: Editorial El Manual Moderno.

-Cenidh. (2013, 3 de Junio). Informe derechos de la niñez, adolescencia y juventudes en el 2013. Cenidh, 8, pp. 11-15.

-Código de la Familia de la República de Nicaragua, Ley No 870 Octubre 2014, Edición 2014

-Código Penal de Nicaragua Mayo 2008.Edicion 2008.

-Colín Sánchez, Guillermo, Derecho Mexicano de Procedimientos Penales, $20^{\mathrm{a}}$ edic. (Y anteriores), ed. Porrúa, México, 2009.

-Constitución Política de Nicaragua, Ley No 330 enero 2000.Edicion 2005

-Cruz C. (2010). Tipifican por primera vez delito de pederastia en México. El Economista, Pp.5-8.

-Fuertes R. (2014, 27 de Agosto). Pedófilos y pederastas: similares pero no iguales. La voz Libre, Salud Mental, 12, p.2.

Krafft-Ebing, R. (2006). Psychopathia sexuales (Psicopatía del sexo). Alemania: Historieta.

Ley No 228, Ley de la Policía Nacional. Managua 23 de Agosto de 1996 Edición de 1996.

-ONU. (1989) Convención sobre los Derechos del niño adaptada y abierta a la firma $y$ adhesión por la Asamblea General en su
Resolución 44/25 de 20 de Noviembre de 1989 entrada en vigor el 2 de septiembre de 1990 de conformidad con el artículo 49.Edicion 1990.

-Percy William A. (1996,20 de Marzo). Pederastia y Pedagogía en la Grecia Arcaica. Urbana and Chicago: University of Illinois Press, pp. 30-35.

-Torres Tópaga, William. Delitos contra la libertad, integridad y formación sexual. 2 da. Colombia. Universidad Externado de Colombia. 2011

\section{Entrevistas}

Pavón A. (2016, 5 de septiembre). Entrevista a Astrid Bracamontes Especialista en Derecho Penal nicaragüense - Docente y Secretaria Académica de la Universidad Politécnica Nicaragüense (UPOLI). Realizada vía Audio

Pavón A. (2016, 5 de Septiembre) Entrevista a la Psicoanalista Tania Bautista - Directora General de Recursos Humanos del Hospital Bertha Calderón Roque. Realizada Vía Audio

Pavón A. (2016, 7 de Septiembre) Entrevista a la Psicóloga Magda López Directora del Departamento de Psicología en el Centro de Salud Altagracia. Realizada Vía audio

Pavón A. (2016, 8 de Septiembre) Entrevista a la Psicóloga María Dolores, Coordinadora del Centro de Mujeres Ixchen, Realizada vía Audio.

Pavón A. (2016, 11 de Septiembre) Entrevista realizada a la Doctora Eveling Bravo Cabrera, Especialista en Derecho Procesal Penal Nicaragüense y Asesora en el Complejo Judicial Central de Managua. Realizada Vía audio. 